mean level more than this in historic times. But in times beyond historical record this restriction does not apply. (These considerations favour the Darwin theory of the origin of coral reefs.)

This astronomical limit appears to be fundamental for geophysics. It asserts that change of level of the ocean of one centimetre is not permitted, unless the day was exceptionally longer in the glacial periods : if the water were removed to this depth and piled up uniformly on ice-caps within $5^{\circ}$ or 350 miles of the poles, the resulting thickness would be only about $130 \mathrm{~cm}$. or 50 inches, and if within $10^{\circ}$ only 12 inches. Within such narrow limits are accumulations of ice now restricted. Sir George Simpson (Nature, April 2) advances the view that recent ice ages may be due largely to change of temperatures of the Atlantic produced by drift of ice in it down into temperate latitudes ; and here question arises as to the permanent source of supply of the ice. A different mode of origin which has found some favour with the writer is the temporary diversion of ocean currents on a large scale by ice piling up in the narrower parts of their channels and so blocking the flow. Both causes might operate. But the recent glacial period on the central Indian plains appears to be a more intractable proposition.

Holywood, Co. Down,

Northern Ireland. April 12.

\section{Maxwell's Equations and the "Flux-Cutting" Principle}

IN NATURE of February 5, Dr. C. V. Drysdale attempts a simplification of Maxwell's electrodynamic equations, on the grounds that these in their original form are difficult to comprehend and to apply, and not completely fundamental. As regards the latter criticism, it must be admitted that Maxwell rarely leaves much scope for improvement in his mathematical statements of physical laws, and so far as the complexity is concerned, many years' lecturing experience have convinced me that the average electrical engineering student of pass degree standard finds little difficulty in understanding and appreciating the concise and elegant expression of the laws of induction, which he has had ample opportunity of testing experimentally in his laboratory work, and usually finds the wider applications, to transmission, skin effect, and metallic reflexion, both easy and interesting.

A closer examination of Dr. Drysdale's argument leaves my opinion of Maxwell's achievement unshaken.

The first modification is introduced rather abruptly with the words: "if we write $j \frac{\partial E}{\partial x}$ for curl $E$ in the equation - curl $E=\dot{B}$. . " It is not easy to see any justification for this suggestion. It is true that the components of the vector curl $E$ may be obtained by applying the differential operator $\mathbf{i} \frac{\partial}{\partial x}+\mathbf{j} \frac{\partial}{\partial y}+\mathbf{k} \frac{\partial}{\partial z}$ in a vectorial manner to the vector $E$, where $\mathbf{i}, \mathbf{j}$ and $\mathbf{k}$ are the unit vectors along the axes $X, Y$ and $Z$, respectively, that is, by de. veloping the determinant

$$
\left|\begin{array}{ccc}
\mathbf{i} & \mathbf{j} & \mathbf{k} \\
\frac{\partial}{\partial x} & \frac{\partial}{\partial y} & \frac{\partial}{\partial z} \\
E_{x} & E_{y} & E_{z}
\end{array}\right|
$$

But here the scalar products $\mathbf{i} \times \mathbf{i}=\mathbf{j} \times \mathbf{j}=\mathbf{k} \times \mathbf{k}=\mathbf{1}$, and the vector products $\mathbf{i} \wedge \mathbf{i}=\mathbf{j} \wedge \mathbf{j}=\mathbf{k} \wedge \mathbf{k}=0$, whereas Dr. Drysdale later asserts that $\mathbf{j}^{2}=-1$, and thus confuses the unit vector $\mathbf{j}$ with the electrical engineer's phase rotating operator and tempts the reader to suspect that the relation $E=j V B$ expresses a quadrature phase difference between $E$ and $B$.

The next step is equally sweeping and illegitimate : it consists in "writing", $\frac{\partial B}{\partial t}=\frac{\partial B}{\partial x} \frac{d x}{d t}$.

It is now obvious, however, that the original equations have been reduced by making some very special assumptions, namely, that $E_{x}=E_{y}=0$, and $B_{x}=B_{z}=0$, leaving

$$
\frac{\partial E_{z}}{\partial x}=\mu \frac{\partial H_{y}}{\partial t} \text { and } \frac{\partial H_{y}}{\partial x}=\varepsilon \frac{\partial E_{z}}{\partial t},
$$

and by this specialization a set of equations has been obtained which ipso facto is less general, that is, less completely fundamental, than the original system.

It is, of course, easy to obtain a simple wave equation from the above two equations, and the solution and discussion would seem fairly simple, but Dr. Drysdale prefers to introduce a magnet which is moved transversely through a non-conducting medium with a velocity $V$, inducing an E.M.F. $E=-j \mu V H$, this E.M.F. in turn inducing a magnetic force $H^{1}=\varepsilon \mu V^{2} H$, etc. It is asserted that thus an electromagnetic wave is generated, though it is not easy to trace any connexion with wave motion in the ordinary sense. It is further pointed out that, as the velocity increases, the fields will increase, unless $H$ is diminished to $H-H^{1}=\left(1-\varepsilon \mu V^{2}\right) H$, and it is concluded that when $1-\varepsilon \mu V^{2}=0, H$ is also 0 . This is tantamount to a statement, that when one factor in a product is 0 , the other factor is also 0 .

The extension of this argument to the case of semiconductors shows an equal disregard for elementary rules of ordinary, partial and total differentiation and leads to yet another curious equation,

$$
\frac{d^{2} E^{1}}{d t^{2}}=V^{2} \frac{\partial^{2} E^{1}}{\partial t^{2}}
$$

Dr. Drysdale's main contention is that flux-cutting is more fundamentally correct, as an E.M.F. inducing agent, than flux variation, but it is well to remember that when the dynamo designer speaks of a conductor, he is at the moment concentrating his attention on one side only of a complete armature coil-if the other side were not there, his dynamo would scarcely be a success. It is, of course, the flux variation through the armature coil which generates the E.M.F. Dr. Drysdale himself admits that, in the case of a transformer, the flux variation principle is correct (and even "more convenient"), and the attempt at saving the flux-cutting principle, by invoking some mysterious and incomprehensible "cutting" by a stationary coil, can scarcely be taken seriously.

Yet Dr. Drysdale claims to have rendered Maxwell's equations more completely fundamental and more intelligible, and urges that his representation "should be adopted". It might well be asked-by whom? If text-book writers were to adopt this kind of argument, it could only add to the confusion.

\section{Welbeck Street, London, W.1.}

No one has a greater admiration for Maxwell's magnificent achievements than I have, and so far from wishing to decry them, my main object has 